\title{
Redes de Sensores Sem Fio em Ambientes Industriais: Estudo de Caso em uma Subestação de Energia Elétrica
}

\author{
Lilian C. de Freitas, Noberto Bramatti, André M. Cavalcante, João C. W. A. Costa, Renato Francês
}

\begin{abstract}
Resumo-Este trabalho apresenta um estudo de caso de redes de sensores sem fio voltado para aplicações em ambientes industriais. Neste estudo, é implementado um sistema de monitoramento o qual é responsável por monitorar medidas de aceleração de uma máquina rotativa (compensador síncrono), instalada em uma subestação de energia elétrica. Fatores como interferência, alcance de transmissão, confiabilidade da comunicação e precisão dos sensores são analisados e discutidos.
\end{abstract}

Palavras-Chave-RSSF, máquinas elétricas, monitoramento industrial.

Abstract-This work presents a case study of wireless sensor networks applied on industrial environments. In such one, an electrical machines monitoring system is implemented, which is responsible for monitoring acceleration measures of a rotative machine (synchronous compensator) installed in an electric power substation. Factors as interference, transmission range, communication reliability and accuracy of the sensors are analyzed and discussed.

Keywords - WSN, electrical machines, industrial monitoring.

\section{INTRODUÇÃO}

Recentes avanços tecnológicos nas comunicações sem fio e na eletrônica têm propiciado o desenvolvimento de redes de baixo custo, baixa potência e de sensores multifuncionais. Este cenário levou à criação das denominadas Redes de Sensores Sem Fio (RSSFs). Essas redes são compostas por minúsculos dispositivos capazes de sensoriar, processar dados, e comunicar-se com outros, através de canais de rádio frequiência [1]-[3]. Tais dispositivos podem monitorar e obter informações sobre fenômenos físicos que seriam difíceis, ou até impossíveis, de serem obtidas utilizando redes convencionais. Dessa forma, RSSFs possuem um vasto potencial de aplicação em diferentes áreas, por exemplo: na área militar, na área médica, na área ambiental, e na área indústrial. [1]-[7].

A aplicação de RSSF em ambientes industriais representa uma classe de aplicações com enormes potenciais e benefícios. Muitas soluções sugerem a eliminação de cabeamento em ambientes industriais, dentre as quais RSSF têm se mostrado uma nova e promissora tecnologia [8],[9]. Sensores são essenciais para automação industrial. Estes provêem uma ligação

Lilian Freitas, Renato Francês, João Costa, Universidade Federal do Pará (UFPA), Laboratório de Eletromagnetismo Aplicado (LEA), Belém, PA, 66.075-900, Brasil. E-mails: \{liliancf, jweyl, rfrances\}@ufpa.br. Tel/Fax: +55 91 3102-7740. Noberto Bramatti, Eletronorte - Centrais Elétricas do Norte do Brasil S/A. E-mail: bramatti@eln.gov.br. André M. Cavalcante, Instituto Nokia de Tecnologia (INdT). E-mail: andre.cavalcante@indt.org.br. Este artigo foi financiado pelo $\mathrm{CNPq}$ vital entre sistemas de controle e o mundo físico. Atualmente, sofisticados hardwares e softwares para sistema de controle estão criando diversas possibilidades de automação na indústria [10]. No entanto, a relação custo-eficiência pode apresentar restrições ao uso de sensores tradicionais (sensores cabeados) em aplicações industriais [11], devido principalmente a custos com cabeamento e com manutenção dos sensores, dificuldades para implantar um sistema cabeado em áreas de difícil acesso, ou com restrições, incompatibilidade de protocolos entre vários tipos de sensores e hardware/software de sistemas de controle, alto custo de instalação e alta taxa de falhas de conectores de cabos. Estes são apenas alguns dos fatores que têm impulsionado a pesquisa de alternativas sem fio. Dessa forma, o desafio que emerge é migrar, integrar e/ou substituir as redes de sensores cabeadas por RSSF.

Neste contexto, este artigo apresenta alguns experimentos referentes à aplicação de RSSF em ambiente industrial, especificamente no monitoramento de uma máquina rotativa em uma subestação de energia elétrica. O mesmo está organizado da seguinte forma: A Seção II descreve brevemente aspectos de redes de sensores sem fio. A Seção III descreve a metodologia adotada na realização do estudo de caso. A Seção IV apresenta os experimentos realizados e os resultados obtidos. As conclusões são feitas na Seção V.

\section{Redes de Sensores Sem Fio}

As RSSFs são projetadas para detectar eventos ou fenômenos físicos, coletar e processar dados, e transmitir as informações sensoriadas para usuários interessados [3]. São compostas por pequenos dispositivos, chamados de nós sensores e por pelo menos um ponto de comunicação, denominado estação base. Os nós sensores podem ser equipados com um ou mais sensores, os quais monitoram o ambiente de acordo com a aplicação a que se destinam. São distribuídos espacialmente em uma determinada área física que se deseja monitorar, e usam enlaces sem fio para realizar tarefas de sensoriamento [5],[6]. RSSF para aplicações industriais devem atender aos seguintes requisitos [11]:

- Qualidade de Serviço (QoS) similar às redes tradicionais;

- Tempo de atraso reduzido (possibilidade de execução em tempo real);

- Confiabilidade e segurança dos dados;

- Coexistência com a estrutura legada;

- Robustez frente às condições ambientais agressivas (por exemplo: interferência). 
Nesse contexto será apresentado um estudo de viabilidade da aplicação de RSSF em um ambiente industrial, através da implementação de testes práticos em tal ambiente, de acordo com a metodologia a seguir.

\section{Metodologia Adotada nos Experimentos}

A metodologia utilizada consistiu na instalação e configuração de uma RSSF, e na realização de testes experimentais nesta rede. Nesta fase, um software de coleta e análise de sinais de vibração, chamado WSensor Networking, foi implementado utilizando a linguagem Labview 7.1 [13]. Este é responsável por receber as medidas de aceleração coletada pelos nós sensores, possibilitando a análise no domínio do tempo e da freqüência. Além disso, em laboratório, foi realizada a calibração dos nós sensores, os testes de precisão destes, os testes de comunicação entre o nó sensor e a estação base e os testes de sensibilidade em freqüência. Este último objetivou analisar a capacidade do sistema de responder satisfatoriamente quando submetido a sinais de diferentes freqüências de aceleração. Em um segundo momento, estando os sensores calibrados e o software de monitoramento desenvolvido, foram realizados testes em um compensador síncrono, em operação na subestação de energia elétrica da empresa Eletronorte, situada em de Vila do Conde, na cidade de Barcarena, no estado do Pará. Estes testes objetivaram, basicamente, analisar o comportamento da RSSF em um ambiente industrial real (sujeito a interferências), e validar os testes realizados no laboratório.

A seguir, é apresentada a descrição dos principais hardwares utilizados neste estudo de caso, objetivando uma melhor compreensão das atividades desenvolvidas.

\section{A. O Compensador Síncrono}

Os compensadores síncronos (ilustrado na Figura 1) são máquinas importantes nos sistemas de transmissão de energia elétrica, têm como função principal manter estável a tensão na linha de transmissão [14],[15]. A falta temporária dessas máquinas pode ocasionar problemas de estabilidade e diminuição da potência transmitida, gerando problemas para empresas concessionárias de energia elétrica, tais como: diminuição de receita, possível escassez de energia e multas aplicadas pela Agência Nacional de Energia Elétrica (ANEEL) [16], em caso de paradas não programadas.

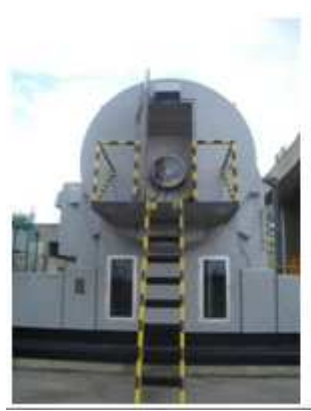

(a) Visão Frontal

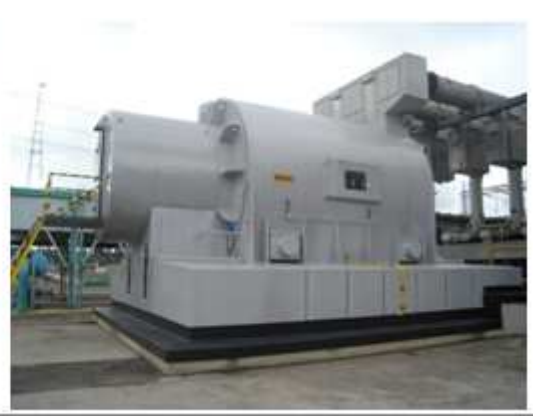

(b) Visão lateral
Fig. 1. Compensador Síncrono
Problemas nos compensadores síncronos demandam normalmente muita manutenção por parte das equipes especializadas, seja do tipo manutenção preditiva, a qual se procura prever os problemas e atuar de imediato nas suas causas; seja do tipo preventiva, em que periodicamente são realizadas intervenções, substituindo peças que se desgastam; ou do tipo corretiva, resultando, geralmente, em intervenções demoradas e de alto custo [14]. Atualmente, a empresa Eletronorte dispõe de um sistema de monitoramento de vibração do compensador síncrono, chamado Vibrocomp [14]. Este se destina a monitorar medidas como vibração do compensador, objetivando a manutenção preditiva. Aumentos na vibração podem indicar a evolução de possíveis problemas mecânicos, geralmente relacionados com desalinhamento, desbalanceamento ou desnivelamento, além de outros problemas específicos em determinados componentes mecânicos, tais como bombas, mancais ou afrouxamento de componentes do estator. $\mathrm{O}$ atual sistema implantado utiliza cabos para interligar os sensores à base de dados, na qual as informações são armazenadas, isto demanda custos adicionais, além de apresentar problemas inerentes a cabeamento, como mau contato nos conectores. Dessa forma, o sistema proposto neste artigo, que consiste no monitoramento através de RSSF, se apresenta como uma solução alternativa de monitoramento industrial, principalmente em casos onde instalar uma infraestrutura com sensores cabeados se torne proibitiva, devido aos problemas já citados.

\section{B. Modelo de nó sensor sem fio}

Na construção da RSSF utilizou-se o modelo de nó sensor sem fio G-Link da empresa MicroStrain Inc. [17]. O nó sensor G-Link, possui um sensor de aceleração capaz de medir aceleração de $\pm 10 \mathrm{~g}$. Este tem uma EPROM pré-programada, assim não é necessária uma maior programação por parte do usuário. Suporta armazenamento onboard de dados de $2 \mathrm{MB}$. É alimentado por uma bateria interna de íon de lítio de 3,6V. Mede $5,8 \mathrm{~cm}$ x $4,3 \mathrm{~cm}$ x $2,6 \mathrm{~cm}$. Cada nó tem um endereço original de 16 bits. A comunicação de rádio-freqüência entre a estação base e os nós sensores é bidirecional, utilizando o padrão de comunicação Zigbee [18], e os nós sensores têm uma taxa programável de amostra de dados. Este modelo de nó sensor especifica um alcance médio de comunicação de 70 metros. Adicionalmente, permite a programação utilizando a Linguagem LabView, a qual é uma linguagem bastante difundida na área de controle e automação industrial. A Figura 2 ilustra o nó sensor G-Link e o nó gateway de comunicação com a estação base, utilizados neste experimento.

\section{Testes Experimentais e Resultados}

Esta seção apresenta os testes realizados em RSSFs no sistema de monitoramento proposto. Como estudo de caso, foi implementado um sistema de monitoramento de máquinas, o qual é responsável por monitorar medidas de aceleração de uma máquina rotativa, chamada compensador síncrono. Fatores como interferência, alcance de transmissão, confiabilidade da comunicação e precisão dos sensores são analisados. 


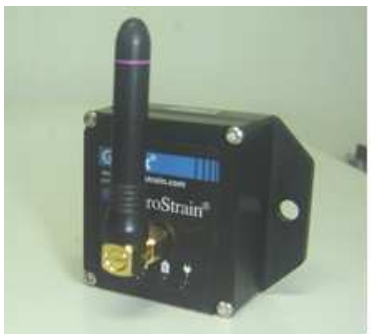

(a) Nó sensor G-Link

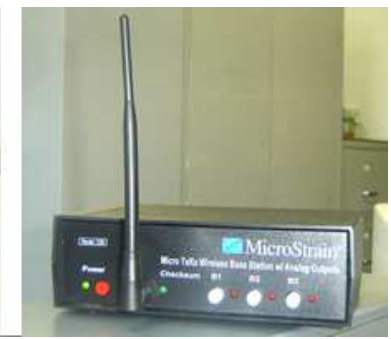

(b) Gateway
Fig. 2. Equipamentos utilizados na montagem da RSSF G-Link

\section{A. Estudo de Precisão dos Sensores}

Ao se pensar em um sistema de monitoramento industrial utilizando RSSF, o primeiro questionamento que surge, está relacionado com a precisão dos nós sensores sem fio. Dessa forma, buscou-se analisar neste estudo se os nós sensores sem fio seriam tão precisos e confiáveis quanto os sensores tradicionais, já utilizados na empresa Eletronorte. Com esse objetivo, realizou-se vários testes comparativos entre os nós sensores sem fio, e os sensores tradicionais, submetendo-os a diferentes sinais de vibrações.

O sensor de aceleração G-Link apresenta faixa de aceleração de $\pm 10 \mathrm{~g}$, com sensibilidade $10 \mathrm{mg}$. A calibração e o teste de precisão do sensor G-Link foi realizada utilizando o equipamento chamado, Shaker DI-813 da empresa Hardy Instruments. Este equipamento possui um gerador de vibração, sendo possível configurar a freqüência e a amplitude da vibração gerada, bem como visualizar sua forma de onda (através do osciloscópio).

O esquema de calibração foi montado fixando o nó sensor G-Link no Shaker DI-813, sendo que o sinal gerado pelo shaker foi visualizado no osciloscópio, conforme mostrado na Figura 3.

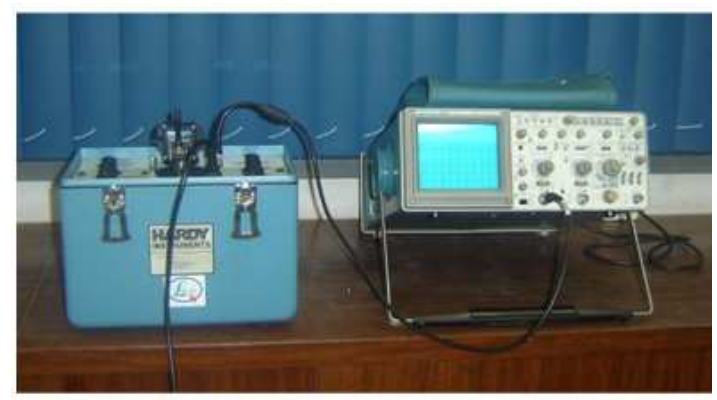

Fig. 3. Esquema de ligação nó sensor, shaker e osciloscópio.

Diferentes valores de freqüência e amplitude de aceleração foram configurados no shaker e enviados pelo nó sensor sem fio para a estação base, localizada a $10 \mathrm{~m}$ de distância.

Os resultados comparativos, entre os valores de aceleração medidos no osciloscópio e os valores recebidos na estação base, mostraram diferenças próximas de 10\%, demonstrando uma precisão satisfatória para aplicação de sistemas de monitoramento implementados na Eletronorte.

\section{B. Teste de Sensibilidade de Freqüência}

Neste teste, avaliou-se a capacidade do sistema de responder satisfatoriamente quando submetido a sinais de diferentes freqüências de aceleração. Este teste foi realizado no Laboratório de Calibração de Transdutores de Vibração (CAME) da empresa Eletronorte, através de um levantamento de resposta em freqüência. Esse levantamento foi implementado pelo método de calibração secundária, caracterizado pela comparação do sensor que está sendo medido, com um padrão de referência, sendo ambos submetidos à mesma vibração. No laboratório CAME, este padrão é rastreado periodicamente a padrões primários no INMETRO (Instituto Nacional de Metrologia, Normalização e Qualidade Industrial), dessa forma garantindo a confiabilidade metrológica das medições. Os resultados mostraram que o nó sensor G-Link apresenta um melhor desempenho quando trabalha na faixa entre $10 \mathrm{~Hz}$ e $90 \mathrm{~Hz}$, pois nesse intervalo a variação de aceleração foi de $\pm 10 \%$ em relação à aceleração padrão. Essa variação foi obtida através da média entre a aceleração em $10 \mathrm{~Hz}$ (que foi $8,5 \mathrm{~m} / \mathrm{s}^{2}$ ) e a aceleração em $90 \mathrm{~Hz}$ (que foi $6,5 \mathrm{~m} / \mathrm{s}^{2}$ ), dessa forma tem-se:

$$
V=\frac{(8,5-6,5)}{2}=1
$$

Onde $V$, que é a variação do nó sensor, corresponde a cerca de $10 \%$ do valor padrão (que é de $9,8 \mathrm{~m} / \mathrm{s}^{2}$ ).

A Figura 4 mostra os resultados obtidos em cada freqüência. Observou-se que a partir da freqüência de $100 \mathrm{~Hz}$, a variação entre o valor do nó sensor e o valor padrão aumentaram, não sendo recomendada a utilização do nó sensor sem fio G-Link nessas freqüências.

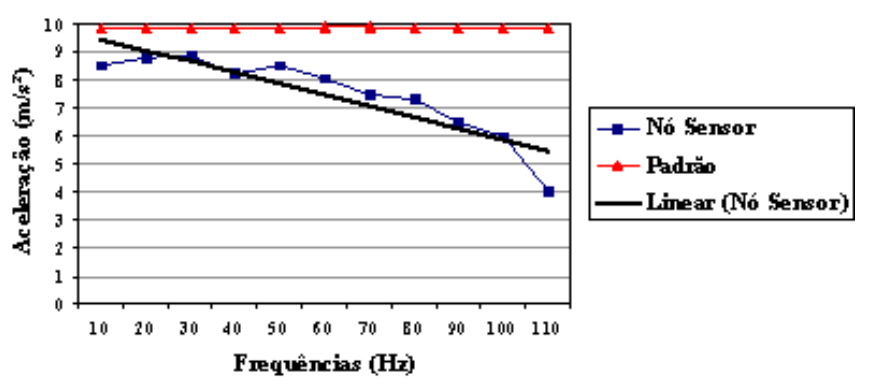

Fig. 4. Resultados do teste de variação da frequiência de aceleração.

Além disso, os resultados evidenciam que o nó sensor G-Link é melhor aplicado em máquinas que trabalhem em uma freqüência fixa, onde seria necessário monitorar apenas a amplitude da aceleração, como é o caso do compensador síncrono utilizado no experimento, que vibra na freqüência 15Hz. Através de medidas coletadas pelo nó sensor, um fator de correção de 1,2 foi aplicado à calibração do nó sensor objetivando uma maior precisão dos sensores. Esse fator de correção foi obtido através da média dos valores de aceleração medido pelo nó sensor, dividido pela média dos valores medidos pelo padrão.

De modo geral, pode-se dizer que os resultados do teste de sensibilidade de freqüência foram satisfatórios, pois o nó sensor G-Link se mostrou eficiente na coleta de sinais de 
aceleração dentro da faixa de $10 \mathrm{~Hz}$ a $90 \mathrm{~Hz}$. Podendo ser este, aplicado no monitoramento de máquinas de baixas freqüências de vibração. Nesse contexto, a RSSF implementada atende aos requisitos de sistemas de monitoramento de compensador síncrono em subestação, no que se refere à sensibilidade de freqüência e precisão dos sensores.

\section{Teste no Compensador Síncrono}

Após os testes de precisão e de sensibilidade de freqüências dos sensores sem fio, realizados em laboratório, efetuou-se os testes em um compensador síncrono (chamado síncrono-1) na subestação da Eletronorte, em Vila do Conde. O objetivo deste teste experimental foi analisar o comportamento da RSSF em um ambiente industrial real, caracterizado pela presença de possíveis elementos interferentes.

Foram realizadas medidas comparativas entre a RSSF e os sensores tradicionais (cabeados), através do analisador e medidor de aceleração, modelo CMVA60 da empresa SKF. Também foram utilizadas medidas comparativas com o sistema Vibrocomp, atualmente implantado. A Figura 5 ilustra este esquema de medição utilizando o medidor e analisador de aceleração SKF.

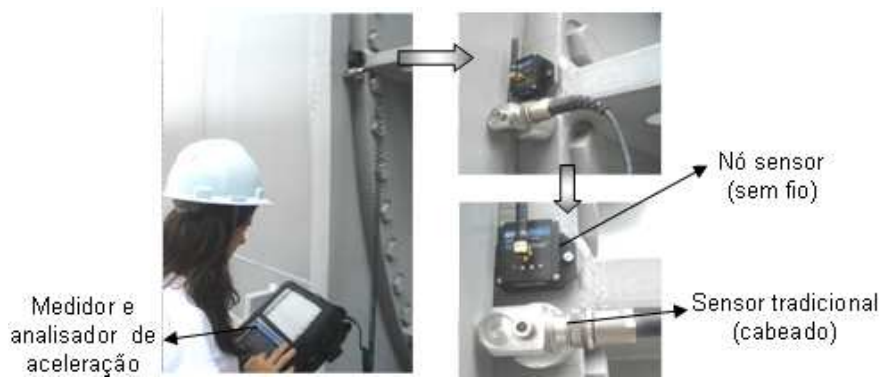

Fig. 5. Esquema de medição utilizando o medidor e analisador de aceleração

Para receber o sinal de aceleração do nó sensor, a estação base foi instalada na sala de operação do compensador síncrono, a cerca de $40 \mathrm{~m}$ de distância do nó sensor. Esta sala é construída em concreto, com portas de ferro e com janelas de vidro.

Inicialmente, acreditou-se que as características da sala e a distância de $40 \mathrm{~m}$ entre a estação base e o nó sensor tornaria proibitiva a realização do teste nesta configuração, mas como este foi o local mais próximo encontrado, insistiu-se nesta configuração para realização dos testes.

A estação base e o nó gateway (receptor do sinal) foram posicionados no final da sala de operação. Colocou-se o nó gateway em cima dos armários para que este pudesse captar mais facilmente o sinal, conforme mostrado na Figura 6.

$\mathrm{Na}$ estação base foi instalado o software WSensor para receber e analisar o sinal de aceleração coletado pelo nó sensor. Utilizou-se uma taxa de 2000 amostras a cada ciclo de trabalho (que é a mesma taxa utilizada pelo sistema Vibrocomp). O nó sensor foi configurado para transmitir a uma potência de $0 \mathrm{dBm}$ e freqüência de $2.48 \mathrm{GHz}$. Dois pontos de medição foram utilizados. Na parte externa do compensador e interna, chamada anel coletor.

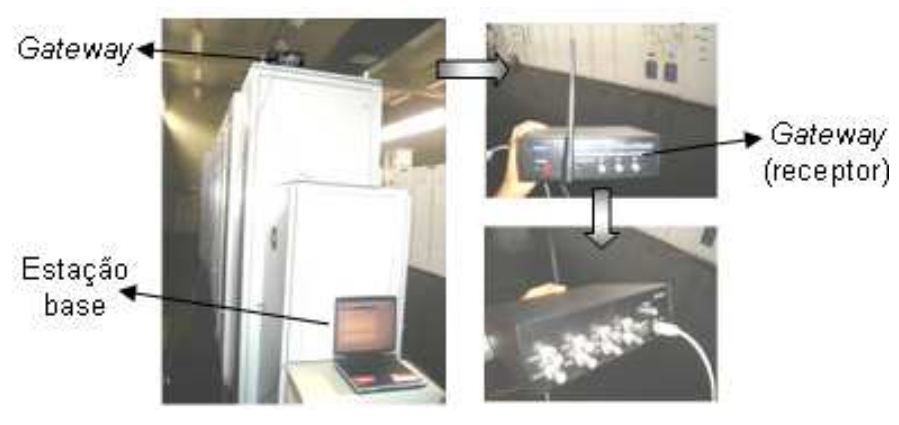

Fig. 6. Conexão com a estação base

Na configuração adotada (estação base dentro da sala de operação do compensador a 40m de distância do nó sensor), foram necessárias algumas mudanças no posicionamento do nó gateway para encontrar a melhor posição de recepção do sinal. Após estes ajustes, a comunicação da RSSF foi estabelecida satisfatoriamente, mesmo diante dos obstáculos (distância, possíveis interferências oriundas de outras máquinas presentes no local, estrutura de concreto da sala de operação, etc.).

Dessa forma, o sinal de aceleração do nó sensor foi medido e analisado. As Figuras 7 e 8 mostram a comparação do sinal de aceleração entre o nó sensor e o sistema Vibrocomp, no tempo e na frequiência, respectivamente.

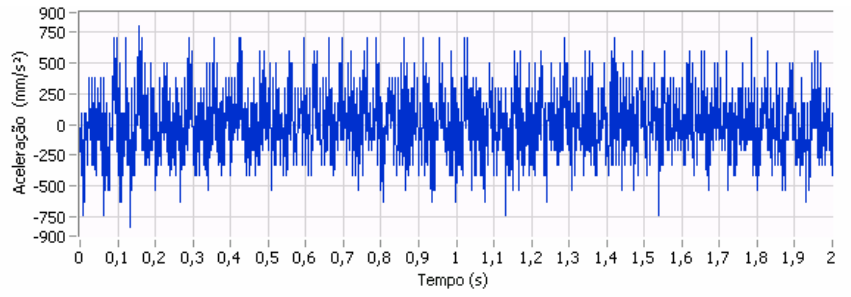

(a) Medidas do nó sensor

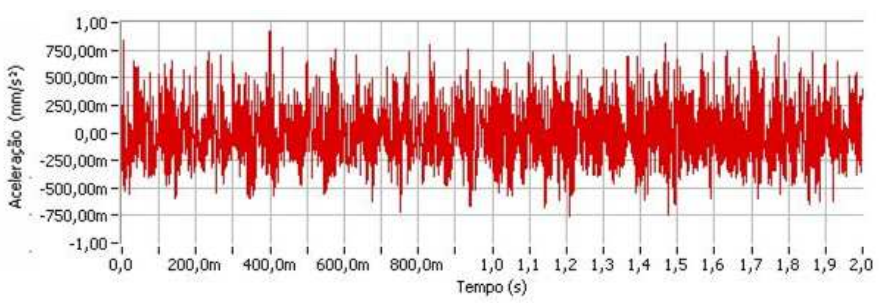

(b) Medidas do sistema Vibrocomp

Fig. 7. Medidas externas ao compensador no domínio do tempo

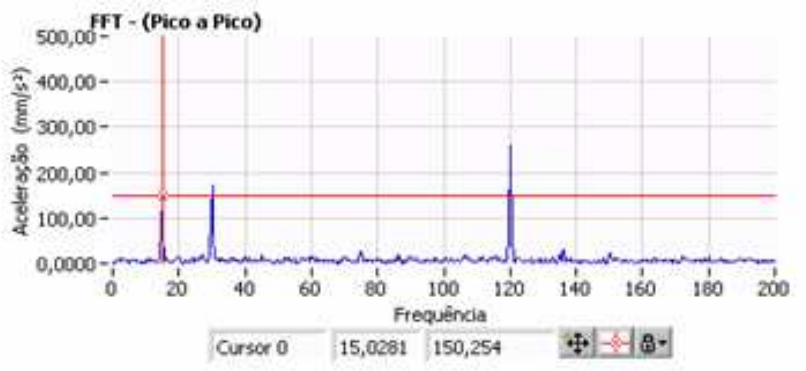

(a) Medidas do nó sensor 


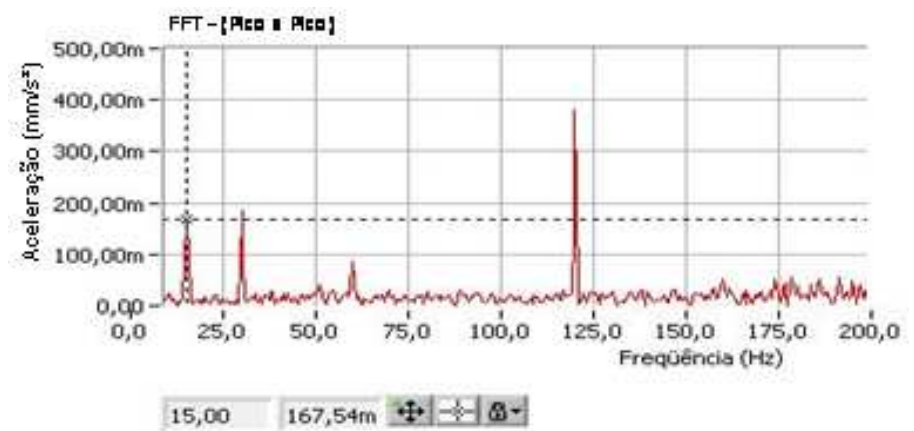

(b) Medidas do sistema Vibrocomp

Fig. 8. Medidas externas ao compensador no domínio da freqüência

Observa-se nos gráficos das Figuras 7 e 8 que o sinal coletado pelo nó sensor e pelo Vibrocomp apresentam características similares. Entretanto, apresentou pequenas diferenças de amplitude comparada com as medidas do Vibrocomp. Observando o gráfico de aceleração, e realizando uma análise na freqüência de $15 \mathrm{~Hz}$, a qual é a freqüência de vibração do compensador síncrono, a aceleração apresentada foi de $150,254 \mathrm{~mm} / s^{2}$, enquanto que a medida pelo sistema Vibrocomp foi $167,54 \mathrm{~mm} / s^{2}$. Essa diferença deve-se a calibração do nó sensor, para se alcançar uma precisão melhor do sinal seria necessário uma nova calibração, considerando especificamente a freqüência de $15 \mathrm{~Hz}$. De qualquer forma esta diferença não influência de forma comprometedora no monitoramento do compensador síncrono.

Além das acelerações presentes na freqüência de $15 \mathrm{~Hz}$, outras acelerações foram observadas na freqüência de $120 \mathrm{~Hz}$, a qual corresponde ao ruído mecânico da máquina. Vale ressaltar que o nó sensor não apresentou ruído elétrico, caracterizado pelo sinal de aceleração na freqüência de $60 \mathrm{~Hz}$.

$\mathrm{O}$ segundo ponto de medição corresponde à parte interna ao compensador síncrono, no anel coletor, conforme mostrado a Figura 9.

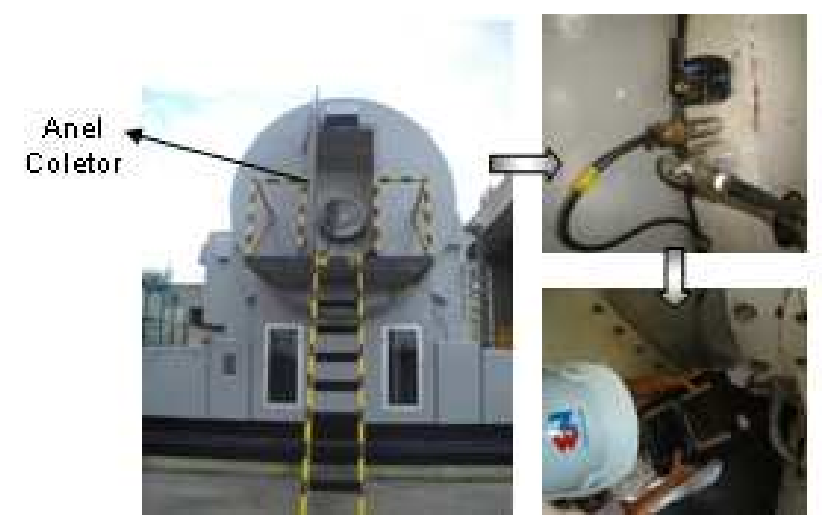

Fig. 9. Posicionamento dos sensores internos ao compensador.

Neste teste, a comunicação entre a estação base e o nó sensor não foi estabelecida, considerando a configuração inicialmente adota, da estação base localizada dentro da sala de operação do compensador. Isto se deve ao fato de que o anel coletor é todo revestido de ferro, dificultando a saída do sinal. Diante disso, uma nova configuração foi adotada posicionando a estação base fora da sala de operação (deixando-a mais próxima do compensador) e mantendo a porta do compensador aberta. Nestas condições foi possível realizar as medições e os resultados são apresentados nas Figuras 10 e 11.

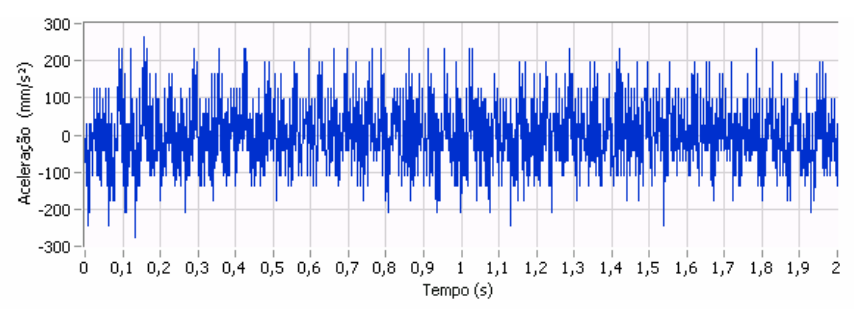

(a) Medidas do nó sensor

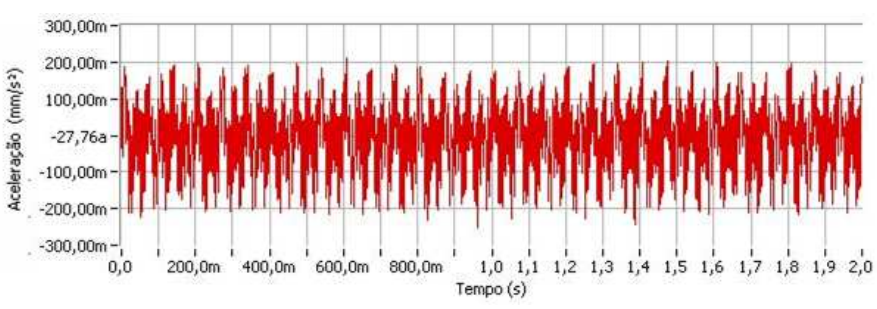

(b) Medidas do sistema Vibrocomp

Fig. 10. Medidas internas ao compensador no domínio do tempo

Observa-se nos gráficos da Figura 10 que o sinal coletado pelo nó sensor e pelo Vibrocomp apresentam as mesmas características no tempo, no entanto, uma análise mais detalhada do sinal pode ser obtida da FFT do sinal, conforme mostrado na Figura 11.

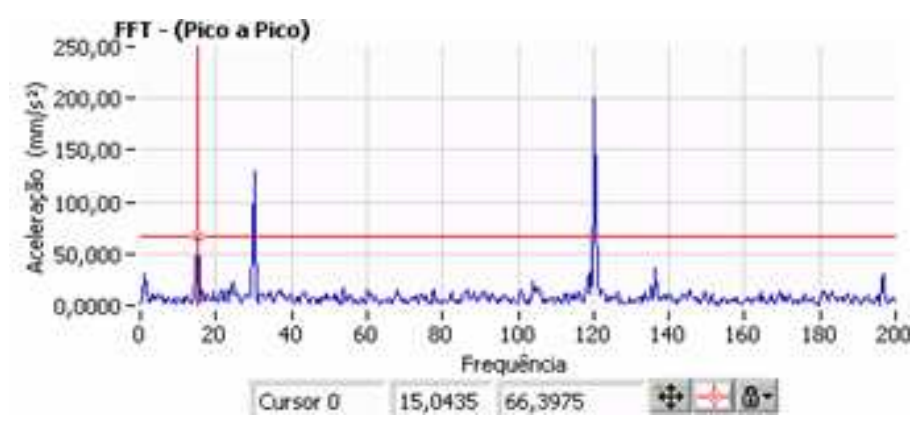

(a) Medidas do nó sensor

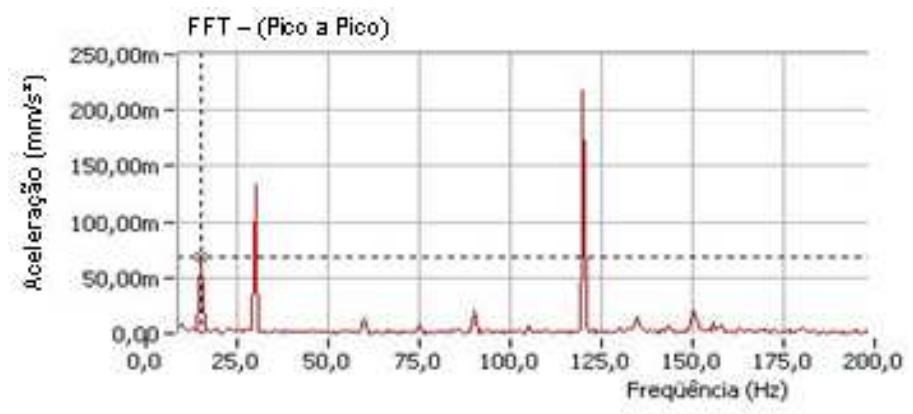

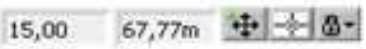

(b) Medidas do sistema Vibrocomp

Fig. 11. Medidas internas do compensador, no domínio da frequência

Novamente, na Figura 11, a comparação entre ambos os gráficos mostra uma pequena diferença nas medidas coletadas. 
No entanto, a relação de variação foi mantida mostrando que a RSSF pode se adequar a um ambiente industrial, pois os valores coletados pelos nós sensores apresentaram bons resultados, e a comunicação na RSSF foi estabelecida com sucesso.

\section{CONClusões}

As RSSF representam, sem dúvida, um ramo de pesquisa com grande potencial de aplicação em diferentes áreas, principalmente na área industrial. Diversas são as vantagens de utilização dessa tecnologia no monitoramento industrial, vantagens estas que envolvem redução de custos com cabeamento, facilidade de implantação de sistemas de monitoramento e flexibilidade dos pontos de medição. Entretanto, várias questões referentes à precisão e tolerância à falhas dos nós sensores são requeridas em tais aplicações, podendo ser estes fatores limitantes para utilização de tal tecnologia. Neste contexto, este artigo realizou diversos testes experimentais em RSSF voltados para aplicações industriais, especificamente no monitoramento de medidas de aceleração de uma máquina rotativa (compensador síncrono), instalada em uma subestação de energia elétrica. Tais experimentos objetivaram avaliar fatores como interferência, alcance de transmissão, confiabilidade da comunicação e precisão dos sensores. Os resultados apresentados (com o uso de modelos de nós sensores GLink da empresa MicroStrain) evidenciaram a viabilidade de implantação desta tecnologia nestes cenários. Entretanto, é importante destacar que RSSF ainda apresentam muitas restrições de aplicabilidade, principalmente no que se refere às interferências. Desse modo, é necessária a análise adequada do ambiente onde a rede será instalada, bem como a escolha dos equipamentos que serão utilizados na montagem da rede e a sua configuração, que envolve local de implantação do nó sensor e da estação base, taxa de transmissão, frequiência de amostragem do sinal, potência de transmissão, entre outras.

\section{AgRADECIMENTOS}

Ao Conselho Nacional de Desenvolvimento Científico e Tecnológico (CNPq) pelo financiamento deste trabalho. Ao Engenherio Anderson Silva pelo esclarecimento de dúvidas.

\section{REFERÊNCIAS}

[1] Haenggi, M., Opportunities and Challenges in Wireless Sensor Networks. In Ilyas, M., Mahgoub, I. (Editors), Handbook of Sensor Networks: Compact Wireless and Wired Sensing Systems, New York: CRC Press, 2005.

[2] Shen, C., Jaikaeo, C., Srisathapornphat, C., Sensor Networks Architecture and Applications. In Ilyas, M. , Mahgoub, I. (Editors), Handbook of Sensor Networks: Compact Wireless and Wired Sensing Systems, New York: CRC Press, 2005.

[3] R. Franck Understanding Smart Sensors, $2^{\mathrm{a}}$ Ed., London: Artech House, 2003.

[4] E. H. Callaway Jr., Wireless Sensor Networks - Architectures and Protocols, New York: AUERBACH, 2004.

[5] Cook, B. W., Lanzisera, S. and Pister, K. S. J., "SoC Issues for RF Smart Dust". Proceedings of the IEEE, volume 94, n 6, June, 2006.

[6] Culler et al., "Overview of Sensor Networks". Published by the IEEE Computer Society, pp. 41-49, August, 2004. Downloaded: www.computer.org.

[7] Bulusu, N., Jha, S., Wireless Sensor Networks - A Systems Perspective, London: Artech House, 2005.
[8] Aakvaag, N., Mathiesen, M., Thonet, G., "Timing and Power Issues in Wireless Sensor Networks - an Industrial Test Case", Proceedings of the 2005 International Conference on Parallel Processing Workshops (ICPPW'05), IEEE.

[9] Jiang, P., Ren, H., Zhang, L., Wang, Z., Xue, A., "Reliable Application of Wireless Sensor Networks in Industrial Process Control", Proceedings of the 6th World Congress on Intelligent Control and Automation, IEEE, June, 2006, Dalian, China.

[10] Evans, J.J., "Wireless Sensor Networks in Electrical Manufacturing". Electrical Insulation Conference and Electrical Manufacturing Expo, 2005. Proceedings Volume, Issue , 23-26 Oct. 2005 Page(s): 460 - 465

[11] Shen, X., Wang, Z., Sun, Y., "Wireless Sensor Netowrks for Industrial Applications". Proceedings of the $5^{\text {th }}$ World Congress on Intelligent Control and Automation, Hangzhou, P.R., China, June, 2004.

[12] Feng, J., Koushanfar, F. and Potkonjak, M., System-Architectures for Sensor Networks Issues, Alternatives, and Directions, IEEE ICCD'02,: VLSI Computers Processors, 226-231, Freiburg, Germany, 2002.

[13] National Instruments Inc., http : //www.ni.com/labview/. Fevereiro, 2007.

[14] Brammati, N., "Desenvolvimento e Implantação de Sistema de Monitoração On-Line de Compensadores Síncronos.”. Dissertação de Mestrado. Departamento de Engenharia Elétrica e de Computação. Universidade Federal do Pará. Belém, PA, Brasil. Dezembro, 1993.

[15] Kiameh, P., Eletrical Equipament Handbook: Troubleshooting and Maintenance. New York: McGraw-Hill companies, 2003.

[16] Agência Nacional de Energia Elétrica. http ://www.aneel.gov.br/. Fevereiro, 2007.

[17] MicroStrain Inc., http : //www.microstrain.com. Janeiro, 2007.

[18] ZigBee Alliance, http ://www.zigbee.org. Janeiro, 2007. 